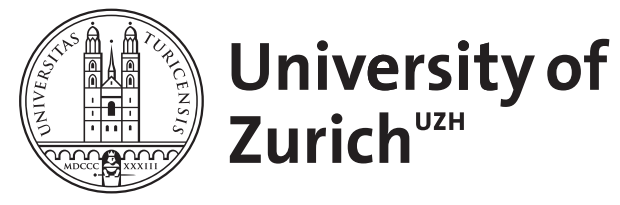
Archive

University of Zurich

University Library

Strickhofstrasse 39

CH-8057 Zurich

www.zora.uzh.ch

Year: 2020

solitudo sine literis (!) exilium est: Die Studierstube zwischen Mußeraum und Musenzimmer

Eigler, Ulrich

DOI: https://doi.org/10.1515/anab-2019-0009

Posted at the Zurich Open Repository and Archive, University of Zurich ZORA URL: https://doi.org/10.5167/uzh-196981

Journal Article

Published Version

Originally published at:

Eigler, Ulrich (2020). solitudo sine literis (!) exilium est: Die Studierstube zwischen Mußeraum und Musenzimmer. Antike und Abendland, 65-66:190-207.

DOI: https://doi.org/10.1515/anab-2019-0009 
UlRich Eigler

\title{
solitudo sine literis (!) exilium est: \\ Die Studierstube zwischen Mußeraum und Musenzimmer*
}

\section{Prolog im Mauerloch}

\author{
Weh! Steck' ich in dem Kerker noch? \\ Verfluchtes dumpfes Mauerloch, \\ Wo selbst das liebe Himmelslicht \\ Trüb durch gemalte Scheiben bricht! \\ Beschränkt von diesem Bücherhauf, \\ Den Würme nagen, Staub bedeckt, \\ Den bis ans hohe Gewölb' hinauf, \\ Ein angeraucht Papier umsteckt; \\ Mit Gläsern, Büchsen rings umstellt, \\ Mit Instrumenten vollgepfropft, \\ Urväter Hausrat drein gestopft - \\ Das ist deine Welt! Das heißt eine Welt! ${ }^{1}$
}

Goethe eröffnet den ersten Teil seines Faust mit einem Spiel der Kontraste. Er stellt den Sonnenstrahlen, die den «Prolog im Himmel» erhellen, jäh die «Nacht» auf Erden gegenüber (33). ${ }^{2}$ Auch erhält die zunächst ausgebreitete überirdische, kosmische Weite des Beginns der Tragödie in der Enge der Studierstube Fausts als Ausgangspunkt für die irdische Handlung einen besonderen Kontrapost.

Jäh fällt wie in einen Guckkasten ${ }^{3}$ der Blick auf Faust, den Protagonisten des Werks. Seine intern fokalisierte monologische Rede präzisiert komplementär zur nullfokalisierten Regieanweisung «NACHT In einem hochgewölbten, engen gotischen Zimmer», im Studierzimmer «FAUST unruhig auf seinem Sessel am Pulte» $(33,334)$ einen Ort, in dessen räumlicher Gedrängtheit und Dichte Fausts Not und Bedrängnis auch im Text

\footnotetext{
* Dieser Beitrag wurde als Vortrag in verschiedenen Versionen im Rahmen des Freiburger SFB 1015 «Muße» sowie an den Universitäten Innsbruck, Basel und Gießen präsentiert, wobei ich für zahlreiche Hinweise und anregende Diskussionen danke. Er war von einer größeren Zahl von Bildern begleitet, die hier nur in beschränkter Auswahl in Schwarz-Weiß wiedergegeben werden können. Gelegentlich wird auf sie hingewiesen. Die folgenden Ausführungen nehmen außerdem zum Teil die Ergebnisse früherer Arbeiten des Verfassers (Eigler 2007, Eigler 2015, Eigler 2017) auf, entwickeln diese aber in neuem Kontext weiter. Für mannigfaltige Hilfe bei Text und Bildern sowie vielfältige Diskussionen und Anregungen danke ich Frau Cornelia Ritter-Schmalz und Frau Anita Harangozó.

${ }^{1}$ Schöne 1977, 34, 398-409. Der Goethetext wird zitiert unter Angabe von Seiten- und Verszahl nach der Ausgabe von Schöne 1977.

2 Im Urfaust fehlt noch dieser Gegensatz, da das Vorspiel im Himmel erst in der späteren Fassung hinzukam. Dazu Schöne 1999, 207.

3 S. dazu die Bühnenzeichnung Goethes in Schöne 1977 Erster Bildteil, Abb. 3.
} 
dreidimensional inszeniert wird. ${ }^{4}$ Raum und Ausstattung verweisen in gleicher Weise auf ihn als Gelehrten, wie er selbst sich auf diese bezieht im Wunsch, sie zu überwinden, ihrer Enge zu entkommen.

Bis an die Decke stapelt sich der «Bücherhauf, [d]en Würme nagen, Staub bedeckt» sowie «angeraucht Papier». Hinzu kommen - außerhalb des zitierten Bereichs - Geräte zu alchemistischen Zwecken (34, 406-408). Die dumpfe Altertümlichkeit komplettieren gemalte Fenster $(34,401) .^{5}$

Damit wird ein weiterer Kontrast hergestellt, der sich aus dem altmodisch-gotischen Raum zu entsprechenden Räumen der Aufführungszeit ergibt, eine Differenz, die Faust durch die Ablehnung seines Zustands akzentuiert. ${ }^{6}$ Die Mischung alchemistischer Geräte mit verstaubtem Bücherfundus verkörpert den Polyhistor-Typ, der bis zur Generation vor Goethe, vom späten 16. bis frühen 18. Jahrhundert, noch aktuell war. ${ }^{7}$ Fausts Studierstube wird somit zur Metapher für ein überholtes Wissenschaftsmodell, die Szene markiert programmatisch die Umbruchsphase von buchorientierter zu empirischer Erforschung von Welt und Natur seit Descartes und Newton. ${ }^{8}$ In tiefer Depression verzweifelt Faust am Leben in diesem nur mäßig hell vorzustellenden Raum, der von ihm selbst als «Kerker» bezeichnet wird. Die ihn umgebenden Bücher und Geräte lehnt er als Ausdruck einer philologischen, d. h. buchorientierten Wissenschaft ab. Hinaus in die Natur drängt es ihn als einen Ort der wahren, empirischen statt autoritätsgestützten Erkenntnis.

Faust tritt unserem Blick an einem zentralen Erinnerungsort der europäischen Kultur entgegen, der als Raum des einsam tätigen Intellektuellen seinen Ursprung im durch Petrarca konstituierten und durch die Petrarcarezeption idealisierten studiolo besitzt, ${ }^{9}$ doch auch einen langen Prozess durchlaufen hat. ${ }^{10}$ In vielen Darstellungen wurde er genutzt, um geistiger Arbeit einen Rahmen zu geben, sie vorstellbar, ja ausstellbar zu machen. Faust jedoch möchte nun aus diesem Raum heraustreten wie saus seiner selbst verschuldeten Unmündigkeit>. Wagner, der Famulus, teilt diese Sicht nicht, sondern will, konservativ und das Alte bejahend, nicht die Nöte seines Meisters sehen. Für ihn entspricht die Studierstube dem positiv konnotierten humanistischen ${ }^{11}$ «Museum» $(38,520)$.

\footnotetext{
${ }^{4}$ Das Bühnenbild entspricht völlig dem Ort des Studierzimmers. Zur Dreidimensionalität und Bildhaftigkeit des Textes s. Pächt 1963, 131.

${ }^{5}$ Goethe wurde maßgeblich durch Rembrandts «Faust in seiner Studierstube» von ca. 1652 (Druckgraphik Walraf-Richartz-Museum Köln) angeregt. Die Identifikation des abgebildeten Gelehrten mit Faust und der Titel gehen auf den niederländischen Kunsthändler Gersin zurück. Vgl. Stierle 2003, 154.

${ }^{6}$ Stierle 2003, 804 charakterisiert zu Recht die Semantik des Begriffs «gotisch» als negativ: «Zur Zeit der Niederschrift ist dies noch eher eine abschätzige Charakterisierung als ein neutraler Stilbegriff». Schöne zu Vers 354 betont, dass «gotisch» «[i]m 18. Jahrhundert für Mittelalterlich-Überlebtes, Unmodernes» (Schöne 1999, 208) steht.

7 S. Schön 1987, 75.

${ }^{8}$ Wie ein Kommentar zur Ablösung des alten Studienambientes durch ein neues, empirisches und nicht buchgestütztes Wissenschaftsideal liest sich die fiktive Geschichte, die Durs Grünbein im Kapitel «Zweite Meditation. Die Schule der Autopsie» erzählt und die er selber (S. 44) als «Heiligenlegende» bezeichnet (Grünbein 2008, 43 f.). Darin lässt er Descartes dem fiktiven Besucher Rembrandt ein seziertes Kalb präsentieren mit den Worten: «Hier, lieber Herr, sehen Sie meine Bibliothek».

9 S. Stierle 2003, 806, der allerdings die Zwischenstufen des Entwicklungsprozesses zwischen Petrarca und Goethes Faust ausblendet.

10 Celenza 2007, 371-391. Grundlegend ist die Arbeit von Liebenwein 1977.

11 Zur Bedeutung des studiolo als Musenraum s. Anderson 1991, 165-186.
} 
Um nun die Spannung zwischen den beiden intern fokalisiert vermittelten Raumwahrnehmungen zu ermessen, müssen wir in die Zeit der Entstehung der Studierstube zurückgehen, da die dort relevanten Elemente sehr wichtig sind, um den gerade erreichten Zustand zu verstehen. Da wir dazu einen großen zeitlichen Bogen schlagen, mögen die folgenden Ausführungen selektiv und bisweilen stark am exemplarischen Einzelfall orientiert anmuten.

Im nächsten Schritt (2.) wollen wir auf Petrarca eingehen, der die Frage der Einsamkeit zusammen mit Büchern in einem besonderen Raum zu seinem Lebensthema gemacht und dabei das Konzept ‘Studierstube` in seinen Grundzügen entwickelt hat. Dies führte zu einer im Zuge der Petrarcarezeption unterschiedlichen Verbreitung und weiteren Ausgestaltung. Eine italienische, frühere ist klar von einer späteren, nordalpinen Rezeption zu unterscheiden. Ein kurzer Blick soll daher im Anschluss (3.) der italienischen Rezeption des studiolo gelten. Daran schließt sich als weiterer Punkt eine ausführlichere Behandlung der spezifisch nordalpinen Verbreitung und in deren Folge eigenen, «deutschen> Tradition der Studierstube an. Hier wird die Bedeutung des Oberrheins und des deutschen Protestantismus hervorzuheben sein (4.). Das von Fausts Famulus Wagner so hervorgehobene Museum als humanistischer Mußeraum und Musenzimmer ist die persistente und weithin anzutreffende Folge dieser Entwicklung. Diese wollen wir in einigen knappen Grundzügen bis zum Ende des 18. Jahrhunderts, bis zur Entstehungszeit von Goethes Faust überblicken (5.). Wir schauen damit auf die Studierstube als Raum, an dem sich zwei wesentliche Umbrüche der europäischen Geistesgeschichte ablesen lassen: die Individualisierung geistiger Arbeit im 14./15. Jahrhundert und ihre Ausdifferenzierung in eine humanistische (Museum) und naturwissenschaftlich orientierte Tradition gegen Ende des 18. Jahrhunderts. Zum Abschluss folgen einige kurze Bemerkungen zur Tradition der Studierstube nach diesem Einschnitt (6.).

\section{Eine Lebensform als Lebensthema: solitudo sine literis exilium bei Petrarca}

In Fausts Studierstube türmen sich die Bücher bis unter die Decke und werden als dominante Attribute des «Mauerlochs» präsentiert, das wie ein «Kerker» den Menschen von der freien Betrachtung der Welt abhält. ${ }^{12}$ Zweifellos steht dieser Raum in der Tradition des seit Petrarca präsenten studiolo, ist dort aber positiv charakterisiert. Die in diesem Raum versammelten Bücher legitimierten ihn als Ort tätiger Muße, der dem Gelehrten eine Heimat schaffte, ihm zu seiner intellektuellen Unabhängigkeit verhalf. Zwei Gelehrtenkonzepte stehen mithin jetzt einander gegenüber, das Petrarcas, des Vertreters eines frühhumanistischen Individualisierungsprozesses, und das des zur Empirie und in die Welt drängenden vormodernen Wissenschaftlers.

Petrarca positioniert sich im ersten Buch seiner in der Abgeschiedenheit von Vaucluse 1346/47 verfassten Schrift De vita solitaria ${ }^{13}$ in der Debatte um die vita activa gegenüber einer vita contemplativa. ${ }^{14}$ Er unterscheidet die Vertreter zweier menschlicher Tätigkeits-

\footnotetext{
${ }^{12}$ Schöne 1999, 202 spricht von Fausts Getriebenheit «nach Weltruhm und Ich-Erweiterung».

13 S. auch Blanchard 2001, 415.

14 S. dazu Enenkel 1990, 376-377.
} 
typen und stellt dem occupatus den von ihm favorisierten otiosus gegenüber. Für letzteren ist nach Petrarcas Auffassung die Verbindung von otium und solitudo notwendig, wobei er sich auf Cicero und Seneca als der Antike entstammende auctoritates stützt. Den Büchern kommt in dieser Lebensform eine besondere Bedeutung zu. Nur durch sie wird die solitudo zu einer echten alternativen Lebensform, die der hektischen Betriebsamkeit im öffentlichen Leben entgegengesetzt ist (Petrarc. De vita solit. 1,3,19): ${ }^{15}$

Equidem solitudo sine literis exilium est, carcer, equuleus; adhibe literas, patria est, libertas, delectatio. Nam de otio illud quidem illud Ciceronis notum: QQuid dulcius otio literato?> [Cic. Tusc. 5,36,105]. Contraque non minus Senece vulgatum: ‘Otium sine litteris mors est et hominis vivi sepultura> [Sen. epist. 82,3].

Ich jedenfalls vertrete die Ansicht, dass die Einsamkeit ohne Bücher nur Exil, Kerker, Folter bedeutet; nimm Bücher dazu, schon ist sie Heimat, Freiheit, Freude. Denn über die Muße kennen wir den Ausspruch Ciceros: ‘Was ist süßer als Muße mit Büchern?> Und als Entsprechung ist jener Ausspruch Senecas nicht weniger verbreitet: «Muße ohne Bücher bedeutet Tod und lebendig begraben sein>.

Gestützt auf seine antiken Gewährsmänner, vertritt Petrarca ein Lebenskonzept, in dem sich Muße und einsame Zurückgezogenheit mit geistiger Tätigkeit verbinden, die in der Beschäftigung mit Büchern besteht und die Muße als Tätigkeit qualifiziert sowie rechtfertigt. Nur der litteratus kann diese in ihren Wurzeln mönchische Lebensweise wählen. ${ }^{16}$

Da sich aber auch eine städtisch-bürgerliche Schicht gebildeter Laien mit einer eigenen Religiosität ${ }^{17}$ entwickelt hatte, die litterati waren, jedoch die vita activa praktizierten, musste Petrarca sein Angebot kontemplativ-einsamen Arbeitens neu ausrichten. ${ }^{18}$ Es ergibt sich dabei das Paradox, dass der Arbeitsraum ein Mußeraum ist, die Bücher in gleicher Weise Teil asketischer Bemühung und solitudo sind, diese aber gleichzeitig angenehm machen. Die Paradoxie hat ihre Wurzel in dem Umstand, dass Petrarca auf das christliche Konzept der Einsamkeit in der Askese zurückgreift, dieses aber explizit mit dem antik-römischen otium-Gedanken verknüpft. Dieser artikulierte gerade im ersten Jahrhundert n. Chr., dem aristokratischen Bedürfnis einer angenehmen Lebensführung entsprechend, die geistige, einsame Tätigkeit als Gegenstück zu öffentlicher Tätigkeit, der eben nicht Attribute von Annehmlichkeit beigelegt werden. ${ }^{19}$ So verbindet sich angenehme Muße mit der Aufgabe, in asketischer Zurückgezogenheit geistig tätig zu sein. Wenngleich Petrarca diese Gedanken programmatisch auf dem Land in Vaucluse entwickelte, war er unbestimmt hinsicht-

15 Zitiert nach Enenkel 1990. Übersetzung vom Verfasser.

${ }_{16}$ Enenkel 1990, 385. Zu der zwar asketischen und mönchischem Arbeiten verwandten Tätigkeit in der solitudo s. aber Eigler 2007. Auch die mönchische Tätigkeit erfuhr bei Petrarca durch Rückgriff auf antike Vorbilder eine besondere Ausformung durch die starke Betonung der Individualität und Unabhängigkeit, wie der deutliche Anklang an Begriffe in Hier. epist. 14,10 (solitudo, carcer) zeigt. S. dazu besonders die Ausführungen von Enenkel 1990, $286 \mathrm{f}$.

17 Mitterauer 2009, 265 betont (mit Literatur) die Zunahme des Lesens im Stillen als Resultat einer gewachsenen Laienfrömmigkeit.

${ }^{18}$ Wichtig ist auch der Einfluss des französischen sowie des päpstlichen Hofes, die als Beispiele einer «erste[n] Spezialisierung des privaten Raumes» von Perrig 2011, 38 genannt werden. Beispiele sind die Oratorien bzw. das estudes Charles V. (1338-1380) und Johannes XXII.

19 Diese Bestrebungen waren auch Teil der kaiserzeitlichen «Flucht ins Private», die sich im Lob der einsamen Freiheit statt öffentlicher Gebundenheit z. B. bei Seneca oder Plinius artikuliert. S. Hindermann 2009, 223-231. 
lich der genauen Gestalt des Rückzugsraums. So verblieb die Option der Übertragbarkeit auch in den städtischen Rahmen, vorausgesetzt die Bücher waren vorhanden und bescheidener Raum stand zur Verfügung, der eventuell durch bukolische Elemente metaphorisch aufgeladen werden konnte.

Petrarca selbst ist nicht konkret, was die Lage der Räumlichkeit betrifft. Verstreute Bemerkungen geben unbestimmte Auskunft. So setzt sich Petrarca von denjenigen ab, die dem Luxus frönen und nicht die Freuden eines bescheidenen Lebens mit Büchern kennen, die ebenso wie ihr Besitzer mit einem kleinen Winkel zufrieden sind. Dies betont er z. B. in einem 1448 in Vaucluse an Giacomo Colonna gerichteten metrischen Brief, den er nach seiner Rückkehr von Rom verfasste (Petrarc. epist. metr. 1,6,180-185). Noch ganz bestimmt ihn die Ablehnung gegenüber der Metropole: ${ }^{20}$

$[\ldots]$ nec gaudia norunt

Nostra, voluptatemque aliam, comites latentes,

Quos mihi de cunctis simul omnia saecula terris

Transmittunt lingua, ingenio, belloque togaque

Illustres nec difficiles, quibus angulus unus

Aedibus in modicis satis est;

Sie kennen meine Freuden nicht, meine andere Lust und die heimlichen Gefährten, die mir alle Zeiten und Länder zusenden und die durch Sprache, Geist und durch Künste in Krieg und Frieden berühmt sind. Sie sind nicht anspruchsvoll, ihnen genügt ein einziger Winkel im einfachen Hause;

Gerade der Aufenthalt in Vaucluse bedeutete eine Phase, in der eine Fülle von Schriften zur einsamen Arbeit mit Büchern entstanden bzw. angefangen wurden. De vita solitaria (begonnen 1346/47) beschäftigte Petrarca aber bis in die letzte Zeit seines Lebens in Arquà, wo er 1371 an der letzten Fassung arbeitete. Es schlossen sich Werke an, die den Eindruck von einem Lebensthema ${ }^{21}$ verstärkten. Alle sind sie der Frage des Rückzugs und der einsamen Kontemplation gewidmet: Bucolicum carmen (1347), De otio religioso (1347; beendet 1357), Secretum (1347; Überarbeitungen 1349, 1353).

Fast wie eine Ausführungsanleitung zu den vorangegangenen Schriften liest sich, was Petrarca im Abschnitt De otio et quiete in De remediis utriusque fortunae 1,21 schreibt. Das Werk ist, nachdem er Vaucluse verlassen hat, 1353-1366 entstanden und als Dialog zwischen der versuchenden Freude und der asketisch kontrollierenden Vernunft gestaltet. Wieder geht es um den persönlichen Raum: ${ }^{22}$

GAUDIUM: Otiosus in thalamo requiesco [...]. RATIO: Labor est materia virtutis et gloriae: hunc qui reiicit, illa reiicit.

FREUDE: In Muße pflege ich der Ruhe in meinem Schlafgemach [...]. VERNUNFT:

Die Arbeit ist das Material für Tugend und Ruhm: ihr absagen heißt ihnen absagen.

Die Vernunft rät vom wollüstigen Genuss der Muße ab und empfiehlt einen Aufenthalt im thalamus, der von Tätigkeit mit Büchern und Gebet erfüllt ist. Petrarca beschreibt hier

\footnotetext{
20 Text und Übersetzung von Schönberger/Schönberger 2004, 336.

21 S. dazu ausführlicher Eigler 2007, Eigler 2015.

22 Text und Übersetzung: Keßler/Schottlaender, München 1988.
} 
ganz im Sinne des Hieronymus die tätige Muße, die zu einer philosophischen Einstellung führt (Petrarc. De remediis 1,21). Es spricht zum Ende die Vernunft: ${ }^{23}$

Non sine causa insomnes industriique laudantur.

$[\ldots]$

At brumalis horae noctis saepe rumpendae, psallendum, studendum, legendum, scribendum, cogitandum, contemplandum, quaerendum, ingenio novi aliquid semper et, quod studio quaesitum fuerit, memoria repetendum.

Nicht ohne Grund lobt man den, der in schlafloser Nacht beharrlich tätig ist. $[\ldots]$

Dagegen sollen die Stunden der Winternacht öfters unterbrochen werden, man soll Musik machen, studieren, lesen, schreiben, nachdenken, Betrachtungen anstellen, immerfort mit dem Geist etwas Neues erwerben und das durch Studium Erworbene im Gedächtnis wiederholen.

Ähnlich äußert er sich in der gleichen Schrift im Kapitel De librorum copia $(1,43)$ über die Bücher, die nur in angemessener Menge für rechte quies sorgen. Das Studium im otium soll zur sinnvollen Verwendung von Büchern führen, die allein zum philosophus macht, nicht zum librarius, der von der Bücherfülle überwältigt wird. ${ }^{24}$

Ein abschreckendes Beispiel bieten Ptolemäus und die Bibliothek von Alexandria, die den Kern des Untergangs schon im Augenblick ihrer Vermehrung enthält. So zeigt der freilich bereits der Petrarcarezeption zuzuordnende - Holzschnitt als Simultanbild den stolzen Moment der Entgegennahme eines Buches durch den König, während im Hintergrund schon ein Diener den vernichtenden Brand vergeblich zu löschen versucht. ${ }^{25}$

Petrarcas Modell des individuell einsamen Studiums mit Büchern in einem der Muße und den Musen gewidmeten Raum macht Schule und wird als studiolo im Quattrocento zu einem häufig begegnenden Phänomen v. a. an Italiens sowie Frankreichs Fürstenhöfen. ${ }^{26}$ Entscheidend ist dabei die unmittelbare Petrarcarezeption.

\section{Petrarca und die 〈Erfindung〉 des Gelebrtenzimmers}

Das Konzept der einsamen Arbeit mit Büchern in einem studiolo ist das Ergebnis eines Prozesses, der mit der unmittelbar nach Petrarcas Tod 1374 einsetzenden Rezeption verknüpft ist und durch diese entscheidend verstärkt wird. Dazu gehört v. a. die Petrarcaikonographie, mit der man sich angesichts seiner vielfältigen Bemerkungen zur Arbeit im

${ }^{23}$ Diese Tätigkeit steht wieder ganz im Gegensatz zur Aktivität des gleich zu Beginn von De vita solitaria charakterisierten Städters, der durch seine materiellen Sorgen geplagt wird und sich selber verliert (Petrarc. De vita solit. 1,2): surgit occupatus infelix habitator urbium nocte media, somno vel suis curis vel clientium vocibus interrupto; sepe etiam lucis metu, sepe nocturnis visis exterritus.

${ }^{24}$ Zum Motiv des übermäßigen Buchbesitzes s. Sen. dial. 9,7.

${ }^{25}$ Der besprochene Holzschnitt zeigt einen Blick auf die Bibliothek von Alexandria mit ihrem Gründer Ptolemaeus im Angesicht einer Brandlegung (Quelle: Francesco Petrarca, Von der Artzney bayder Glück [...], Peter Chalybs/Georg Spalatin, Augsburg 1532 p.56; Staats- und Stadtbibliothek Augsburg, 2 Phil 57.

${ }^{26}$ Minges 1998, 25-29. Wichtig war natürlich auch der Aufenthalt Petrarcas am Hof Karls IV. in Prag. 
eigenen Arbeitsraum mit persönlicher Ausgestaltung ${ }^{27}$ offenbar vorzustellen versuchte, wie denn der Ort von Petrarcas schriftstellerischer Tätigkeit beschaffen war. Petrarca stimulierte also durch seine metaliterarische Bewusstmachung des Schreibens und Lesens in einem bestimmten Raum diese Imaginationen, war aber wohl selbst zu solchen Äußerungen angeregt worden durch analoge Darstellungen wie der Kirchenväterikonographie oder der Evangelistenbilder, ${ }^{28}$ die ihrerseits in der bildlichen Petrarcarezeption wirksam wurden. Auch vollzog sich im Verlauf der Entwicklung der Interieurmalerei der Schritt des Raums vom bloßen Hintergrund zu einem gleichsam mithandelnden Rahmen, der für die Tätigkeit des dargestellten Individuums konstitutiv wurde sowie den Betrachtern eine exemplarische Botschaft übermittelte. ${ }^{29}$ Wie z. B. in der Darstellung Petrarcas im studiolo in der Sala dei giganti in Padua oder am Autorenbild des Darmstädter Petrarca sieht man die italienische Rezeption des Gelehrten, die unmittelbar nach seinem Tode einsetzte und das konkretisierte, was bei Petrarca noch unbestimmt blieb. ${ }^{30}$

Die ostentative Inszenierung einer schreibenden Person hatte wiederum Rückwirkungen auf die traditionellen Autorenbilder, zumal antiker Autoren, in der Buchmalerei wie auch in Holzschnitten, die den neuentstandenen Drucken beigegeben waren. ${ }^{31}$ Als Beispiel für die Aufnahme in der Buchmalerei sei das Autorenbild des Sallust genannt, das gleichsam performativ mit dem Proömium verknüpft ist, welches die Absage des Autors an politisches Engagement und die Ankündigung der schriftstellerischen Tätigkeit enthält (Abb. 1). Das der Schule des Maître du duc de Bedford (1389-1435) entstammende Bild ${ }^{32}$ setzt Sallust als erzählendes Ich wie auch als Autorenbild in eins und präsentiert ihn in einem angedeuteten Gehäus, das ein aufgeklapptes Schreibbrett mit Buch und Griffel wie auch ein Tintenfass enthält. Das Architekturelement schließt das Schreibgeschehen von dem äußeren weltlichen Ereignisbereich ab, in dem - noch ganz in der Tradition des Rittertums - der Knappe mit dem Pferd darauf wartet, dass der Herr sich doch eines Besseren besinnt und zurückkommt. Der Maler adaptiert im Sinne der auf Petrarca zurückgehenden Tradition den Gedanken des otium mit Büchern im angeschlossenen Raum, überhöht also diese Tätigkeit innerhalb des Raums der vita contemplativa gegenüber dem (römischen) negotium des Politikers bzw. (aktuell) dem Dienst des Ritters bzw. der vita activa des geschäftigen Bürgers. Im Mittelpunkt steht die Studierstube des Sallust:

27 Vgl. Eigler 2007, Eigler 2014.

${ }^{28}$ Dazu ausführlicher Pächt 1963, Eigler 2007, Eigler, 2014, Eigler 2015, Stierle 2003. S. auch allgemein Lazzi 2001, 5-17.

${ }^{29}$ Pächt 1963. Hier sind auch Raumdarstellungen des 14. Jahrhunderts zu bedenken wie v. a. die Verkündigungsszene. Vgl. auch Jan van Eycks Anbetungsszene des Kanzlers Philipps des Guten, Nicolas Rolin, von 1437. S. auch Stierle 2003, 143-164.

${ }^{30}$ Die Fresken des heute als Sala dei Giganti bekannten Saals in Padua wurden allerdings im 16. Jahrhundert durch andere ersetzt, das Bild Petrarcas blieb im Zustand des 15. Jahrhunderts erhalten. S. Stierle 2003, 143.

31 Vgl. z. B. das Autorenbild in Herodoti Historiae trad. Lorenzo Valla Venedig 1494 (eine Abbildung findet sich bei Eigler 2007, 22).

32 Eine ausführliche Behandlung der Schule des Maître du duc de Bedford bieten Porcher 1962, 159-170, Hedeman 2006a, 449-464, Hedeman 2006b, 173-196. 


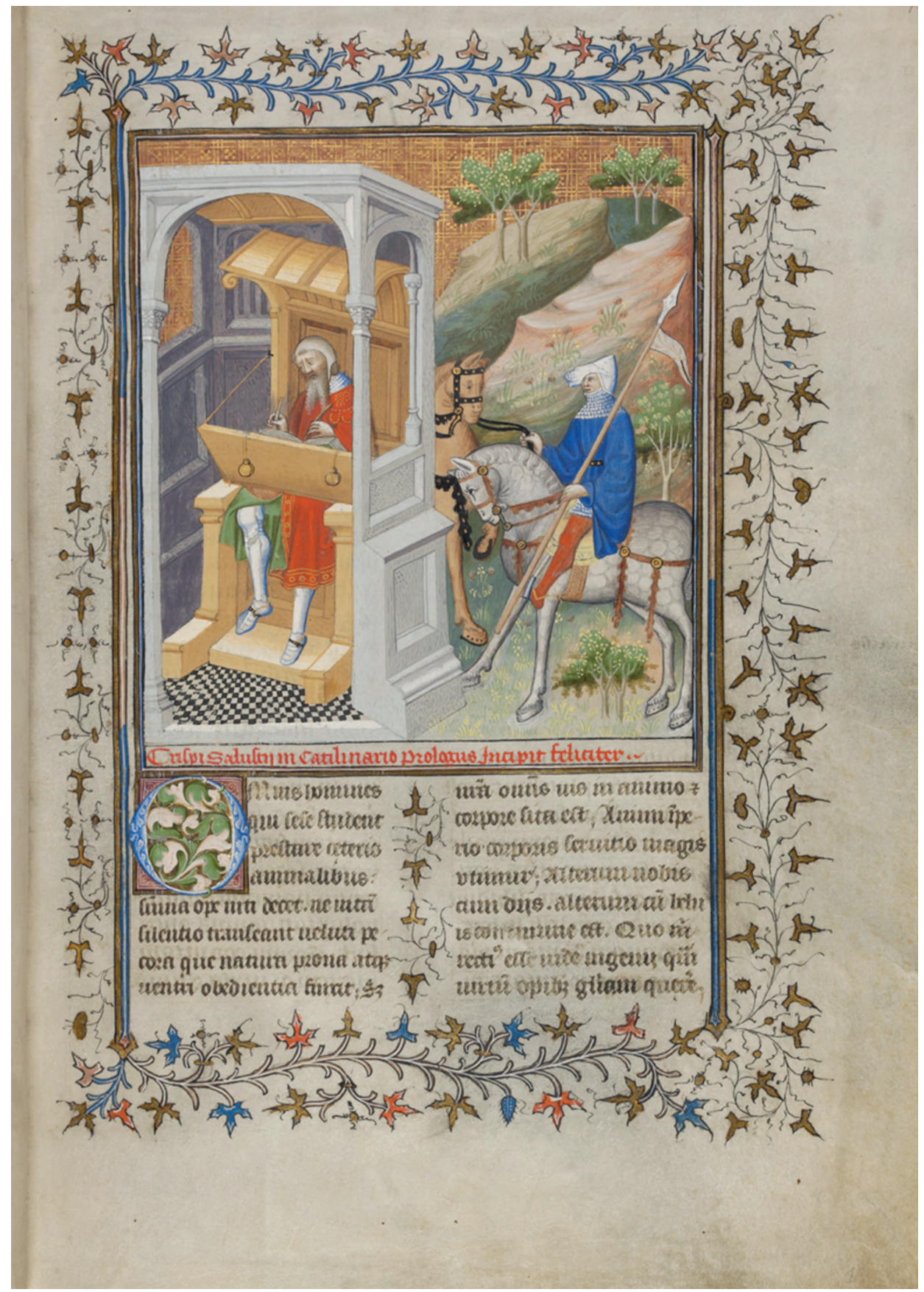

Abb. 1: Sallust, Autorenbild (ca. 1420) (Quelle: Genève, Bibliothèque de Genève, Ms lat. 54, f. 1 (www.e-codices.unifr.ch). Copyright: CC BY-NC 4.0) 
Das reale studiolo hatte in Italien und Frankreich seine Blütezeit im Quattrocento an den Fürstenhöfen, verlor jedoch in beiden Ländern die Funktion des repräsentativen Arbeitsortes und entwickelte sich über einen längeren Zeitraum hin zum reinen «Sammlungsraum», ${ }^{33}$ zum Typus der Kunstkammer im aristokratischen Kontext. Das studiolo im Sinne Petrarcas, als Raum der einsamen Beschäftigung mit wichtigen Büchern, bleibt allerdings auch als ein bürgerliches Phänomen greifbar. ${ }^{34}$ So berichtet Niccolò Macchiavelli in einem Brief an Francesco Vettori vom Zwiegespräch mit den durch Bücher repräsentierten und personifizierten Gelehrten des Altertums. ${ }^{35}$ Er steht damit ganz in der Tradition Petrarcas ${ }^{36}$ (Lettere familiari 216 (10. Dezember 1513)): ${ }^{37}$

Venuto la sera, mi ritorno in casa, et entro nel mio scrittoio; et in su l'uscio mi spoglio quella veste cotidiana, piena di fango e di loto, et mi metto panni reali et curiali; et rivestito condecentemente entro nelle antique corti degli antiqui huomini, dove, da loro ricevuto amorevolmente, mi pasco di quel cibo, che solum è mio, et che io nacqui per lui; dove io non mi vergogno parlare con loro, et domandarli della ragione delle loro actioni; et quelli per loro humanità mi rispondono; et non sento per quattro hore di tempo alcuna noia, sdimenticho ogni affanno, non temo la povertà, non mi sbigottiscie la morte: tutto mi trasferisco in loro.

Wenn der Abend kommt, kehr' ich nach Hause zurück und trete in mein Studierzimmer ein. An der Schwelle entledige ich mich meines Alltagskleides, das mit Schlamm und Kot bedeckt ist und lege königliche höfische Gewänder an. Und, angemessen gekleidet, betrete ich den ehrwürdig alten Hof der Männer des Altertums. Dort werde ich von ihnen liebenswürdig empfangen und nähre mich mit jener Speise, die allein mir zukommt und für die ich geboren bin. Dort schäme ich mich nicht, mit ihnen zu sprechen und sie nach dem Grund ihres Handelns zu fragen. Sie antworten mir in ihrer Menschlichkeit, und vier Stunden lang empfinde ich keine Langeweile. Ich vergesse jeden Kummer, ich fürchte die Armut nicht, der Tod erschreckt mich nicht. Ich lebe ganz in ihnen.

Nördlich der Alpen geht die Rezeption von Petrarcas Muße-Raum mit Büchern eigene Wege. Während die Bildtradition, wie wir gesehen haben, durchaus in Frankreich unter italienischem Einfluss stand und auch Albrecht Dürers «Hieronymus im Gehäus» (1514) diesen erkennen lässt, ${ }^{38}$ führt die besondere Rezeption der Schriften Petrarcas im Norden Ende des 15. Jahrhunderts «verzögert` zur Entwicklung einer eigenen Tradition zur Gelehrtenstube.

${ }^{33}$ Minges 1998, 25 zieht die Grenze wohl ein wenig zu scharf nach dem Quattrocento. Bertilli 2015, 34 betont dagegen den Übergangszustand des 1570 für Francesco I. de’ Medici gestalteten neuen studiolo, das gleichsam "studio alla maniera petrarchesca e il collezionismo di Rudolfo II.», Bibliothek und Kunstkammer miteinander verbindet.

${ }^{34}$ Gerade hier setzt sich offenbar Petrarca durch. Zu weiteren Informationen s. Perrig 2011, 11, der als Beispiel Marsilio Ficino 1989, 117 und 133 (mit Bezug auf Avicenna d. h. Arnold von Villanova) nennt.

35 Eigler 2015, 235.

${ }^{36}$ Nach Liebenwein 1977, 66 braucht es bei Poggio die persönliche und optische Anwesenheit der Autoren als Buch für das Gespräch. Zur Thematik allgemein Brogsitter 1958, 205. Die Entwicklung des Topos von der Lektüre als Gespräch mit den Autoren von Petrarca bis Machiavelli skizziert Bec 1976, 3-17.

37 Text Martelli 1971. Übersetzung Brogsitter 1958, 205-206.

38 Stierle 2003, 143 betont, dass für die Bilder des studiolo das Verhältnis zwischen einem Bildtyp «nordischer und südlicher Malerei im 15. Jahrhundert» zu beachten ist. 


\section{Sebastian Brant und die Neuerfindung der Gelebrtenstube aus dem Geiste Petrarcas}

Bedingt durch den Druck haben Petrarcas Werke eine zwar verzögerte, aber im Ganzen stärkere Rezeption nördlich der Alpen, zumal im Südwesten des Deutschen Reiches, erfahren als in Italien und Frankreich. Dort erfolgte die Wahrnehmung seiner Schriften teils zu seinen Lebzeiten und unmittelbarer, aber überlieferungsbedingt punktueller. ${ }^{39}$ Auch ergab die Wahrnehmung ganzer, seit der Wende zum 16. Jahrhundert im Druck verbreiteter Werkgruppen andere Wirkungen als einzeln rezipierte Handschriften. ${ }^{40}$

Sebastian Brant, der führende Humanist im oberdeutschen Raum, betreute 1496 im Rahmen des sensationellen Versuchs einer Gesamtausgabe von Petrarcas lateinischen Schriften durch die Druckerei Johann Amerbachs auch die Werkgruppe, die im Wesentlichen in Vaucluse entstanden, aber - wie z. B. im Falle von De vita solitaria in späteren Jahren fortgesetzt worden war. Zu den in Vaucluse verfassten und begonnenen Schriften gehörten das nach dem Vorbild von Vergils Hirtendichtungen verfasste Bucolicon carmen, De vita solitaria, De otio religioso, das Secretum und De remediis utriusque fortunae. ${ }^{41}$ Die Titel, von denen ja bereits einige genannt wurden, vereinen die zentralen Werke über die Frage eines sinnvoll gestalteten einsamen Lebens. Der Druck vermittelt suggestiv den Eindruck einer kohärenten Synchronie von Gedanken, die bei Petrarca Äußerungen eines lebenslang betriebenen Nachdenkens waren, dessen diachrone Behandlung keinesfalls südlich der Alpen in vergleichbarer Geschlossenheit rezipiert werden konnte. Die Möglichkeit der Verbreitung durch den Druck traf zudem nördlich der Alpen auf ein Publikum, das bereits durch Gedanken und Bilder zum Thema des einsamen Arbeitens, ${ }^{42}$ die zweifellos aus Italien kamen, vorbereitet war. Der von Sebastian Brant betreute Amerbach-Druck wurde daher nicht nur am Oberrhein, sondern europaweit zu einem Bestseller. Dies belegen die heute noch erhaltenen 393 Exemplare. ${ }^{43}$

Dass die einzelnen Schriften systematisch zusammengestellt wurden, gibt Sebastian Brant durch ein 20 Verse umfassendes programmatisches Einleitungsgedicht De Francisci Petrarchae laude et praestantia ${ }^{44} \mathrm{zu}$ erkennen, mit dem er gleichsam eine exemplarische Vita entwirft, wie man in den drei Schritten ingenium - conversio - vita solitaria zum sinnerfüllten Leben in asketischer Einsamkeit gelangt. Damit nimmt er den Gedanken Petrarcas aus

39 Schottlaender 1988, 24f. hebt die große Bedeutung von Petrarcas persönlichen Verbindungen für die Transmission seiner Werke, aber auch die dadurch begrenzte Rezeption seiner Werke hervor. Er beobachtet dies gerade für die hier relevanten Schriften zum Themenkreis des einsam-asketischen Lebens, wie De vita solitaria, De otio religioso und das Secretum. Allerdings erlebte De remediis utriusque fortunae durch das Interesse am Hof Karls IV. seit 1362 bzw. 1366 eine besondere Verbreitung. Bemerkenswert ist der Schwerpunkt im Deutschen Reich, der auch durch 13 Übersetzungen ins Deutsche dokumentiert wird (Petrarca, 1988, 36).

40 Gerade am Ende des Quattrocento scheint die Petrarcarezeption südlich der Alpen weniger bedeutend als im Norden geworden zu sein. S. besonders Geiß 2002, 101-108 und Karnein 1988, 159-186.

${ }^{41}$ Zum Folgenden allgemein Eigler 2015.

${ }^{42}$ Das Interesse war bereits durch Bilder mit der Petrarcaikonographie, sicher aber auch durch die weite Verbreitung von De remediis utriusque fortunae im Deutschen Reich geweckt.

43 Eigler 2015, 85.

${ }^{44}$ Das Gedicht befindet sich auf Blatt 1b des hier zugrunde gelegten, ursprünglich in Passau befindlichen Drucks (UB Basel UBH DH II). Es ist gedruckt in Schnur 1978, 14. 
De vita solitaria auf, dass es eines bestimmten ingenium bedarf, um zum Ziel zu gelangen. ${ }^{45}$ Verkürzend spitzt Brant die Vita in der Bemerkung zu, dass Petrarca nur in Einsamkeit seine großen literarischen Leistungen zu vollbringen vermochte (11-13): ${ }^{46}$

proinde sibi obtinuit famamque decusque perenne,

et fieri meruit carior inde deo.

illic solus enim tot digna volumina scripsit, $[\ldots]$

Dadurch erwarb er sich Ruhm und schuf sich ewige Zierde, und er wurde mit Fug teurer dem Gotte dadurch.

Ganz allein schrieb dort er so viele vortreffliche Bände,

$[\ldots]$

Mit tot digna volumina deutet Brant auf die im vorgelegten Band versammelten Schriften. Petrarcas solitudo cum literis erlebt als Modell ungestörter einsamer Arbeit mit Büchern nördlich der Alpen damit eine besondere Rezeption. Den großen Anteil Sebastian Brants daran veranschaulicht ein vor 1521 entworfener Holzschnitt, den Sebastian Brant für den Druck der bereits oben behandelten deutschen Übersetzung von De remediis utriusque fortunae zur Illustration der Vita Petrarcae gestaltete (Abb. 2). ${ }^{47}$ Wieder stellt er den Aufenthalt in Vaucluse in den Mittelpunkt und präsentiert im Stil des Autorenbilds einen Gelehrten im studiolo bzw. 〈Gehäus〉 als dessen Ausdruck. Dies belegt, wie schnell der Schritt vom allgemeinen Einsamkeitskonzept zu der Arbeit im Studierzimmer als idealer Lebensform für den Gelehrten sich vollzieht. Die Allgemeinheit des Konzepts wird dadurch noch unterstrichen, dass Petrarca auf dem Holzschnitt die Züge Sebastian Brants trägt. Mit dieser Selbsteinschreibung hat Brant für sich und seinen Zeitgenossen das einsame Leben als Gelehrtenideal übernommen. Gerade am Oberrhein sind schnell die Auswirkungen zu spüren.

\section{Mußeraum und Musenzimmer: Die deutsche Karriere der Studierstube}

Die von Sebastian Brant betreuten und, wie im Falle der Remedia, attraktiv durch Holzschnitte gestalteten Petrarca-Drucke trafen auf ein Umfeld, das für die in diesen Schriften enthaltenen Gedanken bereits sensibilisiert war und schnell reagierte. Besonders das Elsass mit seinem bis in die Schweiz reichenden engen humanistischen Netzwerk wurde stark durch den in geographischer Nähe entstandenen Basler Petrarca-Druck beeinflusst. ${ }^{48}$

${ }^{45}$ Geiß 2002, 85, der darauf hinweist, dass Brant hier «Petrarca als poeta christianus vorstellt». Ähnlich enthält Brants Darstellung auch die Werbung für ein bestimmtes Modell einer christlichen intellektuellen Existenz, indem er das nur in der Askese legitime Motiv der Einsamkeit mit der Lebensform des christlichen Dichters bzw. Autors verbindet.

${ }^{46}$ Text und Übersetzung Schnur 1978, 14.

${ }^{47}$ Der Holzschnitt illustriert das am Anfang beigefügte «Francisci Petrarchae leben auff das kürzest begryffen». Brant lieferte die Skizzen für den unbekannten Holzschnittkünstler wohl zwischen 1516 und seinem Todesjahr 1521. Vgl. dazu Petrarca, 1988, 40 f.

${ }^{48}$ Die normative Vermittlung des durch Petrarca verkörperten humanistischen Studienideals ist v. a. in Humanistenviten zu erkennen, wie z. B. in der von Beatus Rhenanus und Jakob Wimpfeling verfassten Lebensbeschreibung Geiler von Kaysersbergs. S. dazu Eigler 2015, 86. 


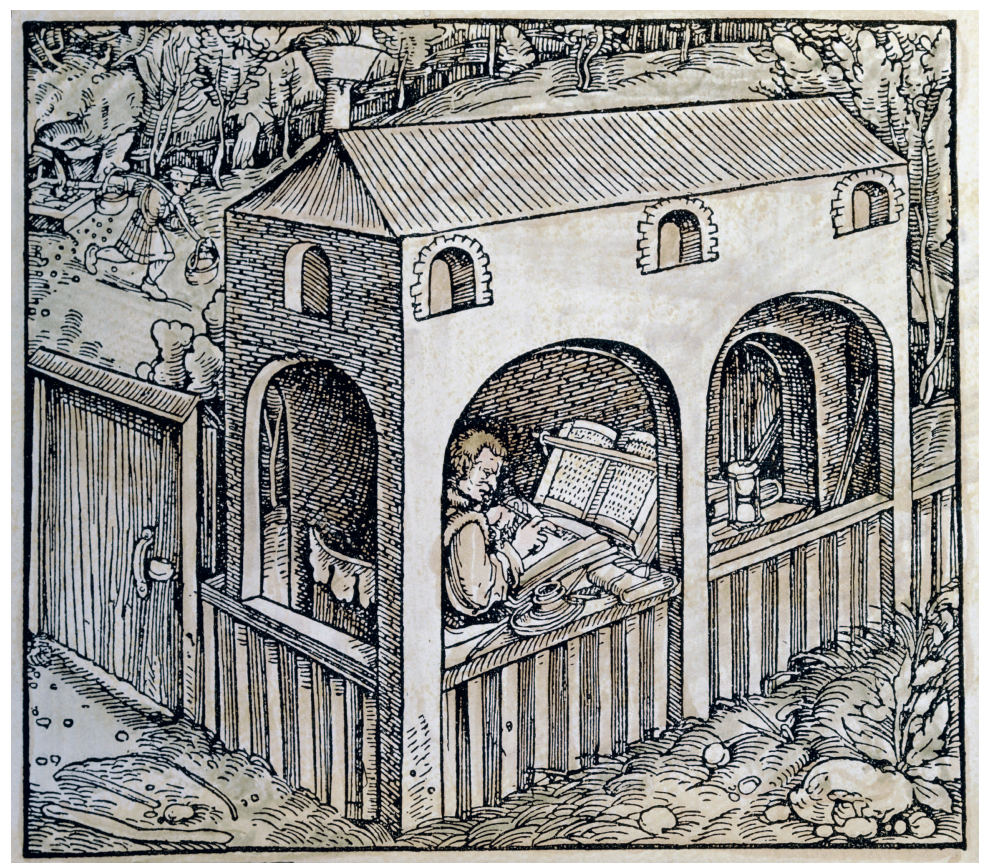

Abb. 2: Petrarca im Gehäus, mit den Zügen Sebastian Brants ausgestattet (Quelle: Francesco Petrarca, Von der Artzney bayder Glück [...], Peter Chalybs/Georg Spalatin, Augsburg 1532. Copyright: INTERFOTO/Alamy Stock Foto).

So steht auch die positive Darstellung der von Beatus Rhenanus (1485-1547) gewählten einsamen Arbeit im Studierzimmer (bibliotheca) in dieser Tradition, die für Menschen mit den entsprechenden Voraussetzungen (natura) die Lebensform der solitudo cum literis nahelegte. So betont auch Iohannes Sturm in seiner Vita des Beatus Rhenanus dessen bescheidenes Leben zu Hause, im elsässischen Schlettstadt, und in seiner Studierstube: ${ }^{49}$

Existimavit naturae et conscientiae suae conveniens esse, domi privatim et temperanter nullo fastu, nullis contentionibus vivere, in sua bibliotheca versari, convivia non magnifice domi instruere, sed ea cum amicis per intervalla concelebrare.

Er war der Ansicht, dass es seinem Wesen und seiner Überzeugung angemessen sei, zu Hause allein und maßvoll ohne Ambitionen und ohne Streit zu leben, in seiner Studierstube sich aufzuhalten, zu Hause keine Gastmähler großartig auszurichten, sondern diese nur gelegentlich mit Freunden abzuhalten.

Das Modell des Petrarca wird freilich nicht nur in Schlettstadt, sondern auch für den 1531 gefallenen Zürcher Reformator Zwingli durch seinen Nachfolger Heinrich Bullinger (15041575) in Zürich in Anspruch genommen. ${ }^{50}$ Die entsprechende Illustration im Kopienband

\footnotetext{
49 Text zitiert nach der Ausgabe von Horawitz/Hartfelder, 1886, 8. Übersetzung vom Verfasser.

50 Auf Petrarca weist eindeutig die Katze zu Füßen des studierenden Reformators. Zu dieser ikonographischen Besonderheit s. Aurnhammer 2005, 18-38.
} 
zur zürcherischen Kirchen- und Reformationsgeschichte von 1605/1606 zeigt die Persistenz des Vorbilds und umspannt die Zeit von Zwingli über seinen «Biographen〉 Bullinger bis zum Beginn des 17. Jahrhunderts. Wir sehen Zwingli in einem Arbeitszimmer mit Butzenscheiben, links und rechts befinden sich wohlaufgeräumte Bücherregale. Der Reformator selbst sitzt, eine Katze zu seinen Füßen, an seinem bestens ausgestatteten Pult. ${ }^{51}$

Die zum Ausdruck gebrachte Vorbildlichkeit für die Gestaltung eines Gelehrtenlebens wird durch die von Bullinger verfasste Studienvorgabe (Studiorum ratio 32) unterstrichen. ${ }^{52}$ Sie illustriert, wie gerade in reformiert-protestantischen Kreisen das studiolo Petrarcas fortlebt: ${ }^{53}$

Eligendus est autem et locus ad studia aptus, qui a turbis et clamoribus sit alienus, siquidem verum est, quod aiunt: Musas gaudere solitudine. Hinc quoque crediderim fabulis proditum esse Musas inhabitare sylvas, montes et virentia prata. Itaque debet studiorum locus situ quoque esse amenus ac prospectu ventorumque et aeris proflatu saluberrimus. At intus omnia compta et munda erunt, queque suum locum occupabunt; nihil indecore, nihil iacebit dispersum indigne.

Es ist aber auch ein Ort zu wählen, der für Studien geeignet ist, der von Unruhe und Lärm geschieden ist, sofern es wahr ist, was man sagt: dass die Musen sich der Einsamkeit freuen. Daher kommt es, möchte man glauben, dass man in den Dichtungen geäußert hat, die Musen bewohnten die Wälder, die Berge und die grünenden Wiesen. So soll der Ort, der den Studien dient, auch seiner Lage und der Aussicht nach angenehm und hinsichtlich der Winde und Luftströmungen gesund sein. Und inwendig soll alles schön geordnet und reinlich sein, alles soll seinen bestimmten Platz innehaben, nichts soll unschicklich, nichts unordentlich da oder dort herumliegen.

Zur dauerhaften Kanonisierung von Petrarcas Studierstube seit dem 16. Jahrhundert zumal im Deutschen Reich - trugen auch emblematische Werke wie der Emblematum liber des Andrea Alciato (1492-1550) bei. Ihm war eine große Wirkung im deutschen Südwesten und schließlich in ganz Europa seit dem ersten Erscheinen einer lateinischen Version 1529 in Basel beschieden. Ihr schloss sich eine mit Holzschnitten illustrierte von Konrad Peutinger (1465-1547) betreute Ausgabe an. In aller Säuberlichkeit sitzt der Gelehrte in seiner Studierstube, die in großer Ordnung die Attribute stiller Lese- und Schreibtätigkeit enthält und dem Bewohner solitudo sowie silentium gewährt, aber auch auferlegt. Eine derartige solitudo cum literis hat nichts gemeinsam mit dem Verzweiflungsort, in dem sich Goethes Faust zu befinden wähnt. Vielmehr schafft sie dem Gelehrten die Gelegenheit, durch Stille zur sapientia zu finden. Dies bringt ein beigegebener Holzschnitt des in säuberlicher Stille arbeitenden Gelehrten zum Ausdruck, erläutert durch ein Distichon: ${ }^{54}$

51 Zwingli in seinem Arbeitszimmer von Heinrich Thomann (Heinrich Bullinger u.a., Kopienband zur zürcherischen Kirchen- und Reformationsgeschichte, Zürich, 1605-1606; Zentralbibliothek Zürich, Ms B 316, f. 231v., http://dx.doi.org/10.7891/e-manuscripta-18901).

52 Text und Übersetzung nach Stotz 1987. S. auch Eigler, 2017.

${ }^{53}$ Ein interessantes Selbstzeugnis bietet auch der Basler Felix Platter, der die Ausgestaltung seines «studiol» in Montpellier beschreibt: Platter 1976, 173.

${ }^{54}$ Ich nehme Bezug auf ein Bild der Paduaner Ausgabe von 1621 (Quelle: Emblemata XI 1621 Padua No. 11 (Alciato)). Übersetzung vom Verfasser. 
Cum tacet, haud quicquam differt sapientibus amens:

Stultitiae est index linguaque voxque suae.

Ergo premat labias, digitoque silentia signet,

Et sese Pharium vertat in Harpocratem.

Wenn er schweigt, trennt nichts den Toren vom Weisen:

Seine Einfalt verrät allein Sprache und Stimme

Soll er also die Lippen verschließen und mit dem Finger schreiben, was still ist, Und sich wandeln in Harpokrates ${ }^{55}$ aus Alexandria.

Die Wirkmächtigkeit der Arbeitsstube dieses Typs unterstreicht auch seine prominente Präsenz im 1658 erstmals gedruckten Orbis rerum sensualium pictus des böhmischen Pädagogen Amos Comenius, einem europaweit verwendeten Schulbuch (Abb. 3). Auch Goethe hatte es benutzt. Wie er selbst in «Dichtung und Wahrheit» schreibt, hatten sich die Bilder ihm eingeprägt. ${ }^{56}$

Comenius präsentiert auf Latein und Deutsch das «Muséum», das «Musenzimmer». Der Raum zeichnet sich durch die bekannte Ordnung aus, die durch die Zuteilung bestimmter Verweiszahlen für die einzelnen Gegenstände noch unterstrichen wird. Dies ist nicht der «Studierkobel» ${ }^{57}$ des Polyhistors, das dunkle Gelass für finstere Künste, sondern der helle Raum ruhiger Erkenntnis in Einsamkeit. Bücher bestimmen ihn wie auch in der Darstellung des im Studium begriffenen Zwingli oder im Emblema Alciatis in sauberer Aufstellung, nicht in bedrückender Häufung. Diese nimmt allerdings Faust wahr und begreift den Ort nicht als Inspirationsraum und die Bücher nicht als Freunde. Faust lehnt damit das Gelehrtenmodell der Arbeit in der Studierstube als überholt ab. Allein der konservative Wagner sieht aus einer alternativen Perspektive in Fausts Stube noch das Museum, den Musenort der Tradition seit Petrarca. Wenn Goethe Wagner vom Museum sprechen lässt, hat er also eine klare Vorstellung vor Augen, die für uns die gesamte Entwicklung dieses wichtigen Ortes offenlegt. Es handelt sich nicht nur um «ein Zitatschnitzel also", wie Schöne mit anderen Kommentatoren meint. ${ }^{58}$ Wagner charakterisiert aus seiner Sicht Fausts Raum positiv und voll Verehrung für das etablierte weltabgewandte Museum des Gelehrten, ${ }^{59}$ das otium cum literis garantiert.

${ }_{55}$ Harpokrates ist der Sohn der Isis.

56 «Die Alten hatten selbst noch kindliche Gesinnungen und fanden es bequem, ihre eigene Bildung der Nachkommenschaft mitzuteilen. Außer dem Orbis pictus des Amos Comenius kam uns kein Buch dieser Art in die Hände; aber die große Foliobibel, mit Kupfern von Merian, ward häufig von uns durchblättert [...].» Text zitiert aus der Hamburger Ausgabe, Goethe (Trunz) 1955, 35.

57 Zitiert nach Schön 1987, 75.

58 Schöne 1999, 221 zu Vers 530. Natürlich erinnert die Aussage Wagners («Ach! wenn man so in sein $\mathrm{Mu}-$ seum gebannt ist, / und sieht die Welt kaum einen Feiertag [...].») an den auch für Spinozas Lebensweise angewandten Topos, wie ihn 1672 Nikolaus von Greiffenkrantz mit Blick auf Spinoza verwendet: Sibi soli vivere videbatur, semper solitarius et quasi in museo suo sepultus (zitiert nach Fischer 2015,182). Hierbei handelt es sich allenfalls um eine kritische Ansicht zur Lebensweise Spinozas, nicht um die Ablehnung der Studierstube.

${ }^{59}$ Der Typus der Gelehrtenbibliothek von Medizinern, Philosophen und Juristen (wie Faust), der neben Büchern auch Instrumente, Naturalien u. a. umfasste, wird in einem Forschungsverbund Marbach/Weimar/Wolfenbüttel erforscht: http://www.hab.de/de/home/wissenschaft/forschungsprofil-und-projekte/ fruehneuzeitliche-gelehrtenbibliotheken.html (Zugriff: 3. 4. 20). 


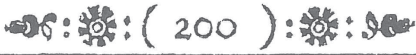

$X C V I I I$.

Muféum. Das Runtitginnter.

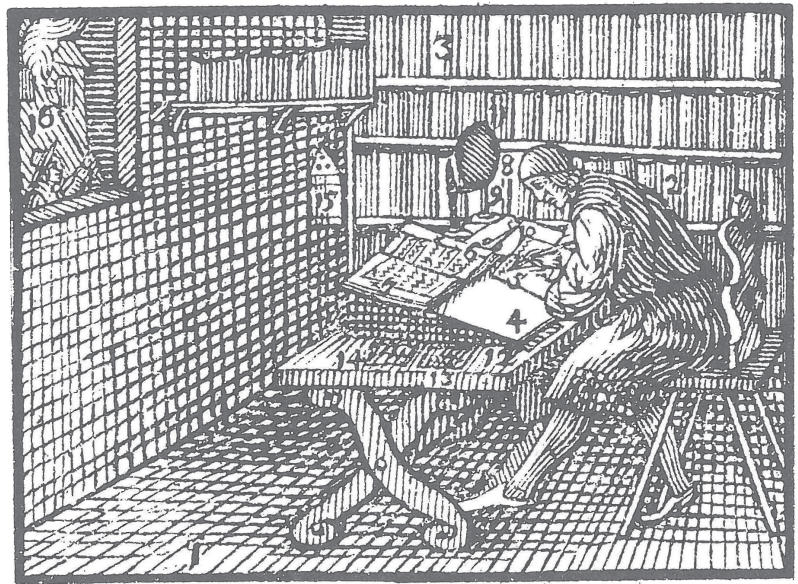

Marférns I efl locus, ubi Studiofus , 2 fecretus ab hominibus, folus feder, Studiis deditus. dum leekitat Libros, 3 quos penes le fuper Pluteum 4 exponit, \& ex illis in Manuale s luum optima quæć; excerpit, aut in illis
Das MTu[enzinmer iftein Drt) ([Gtudont] z wo der Kunfliebende abgefondertwondendeutem alleine fither ;

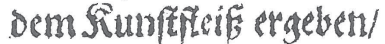
indem er tifer 25 úd)er / 3 weldye et neben fits auf Dem pult 4

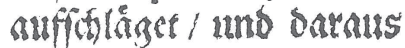
in fén Bandoud ; das baftermigridines/ oder darinnar. 


\section{5. "Wie wird es mir im Busen belle»: otium cum literis bei Faust}

Goethe hat die dramatische Einführung seines Faust an einem zentralen Ort der europäischen Kulturgeschichte platziert, der nach wie vor große Geltung besitzt und in unterschiedlicher Weise die Diskussion um einen richtigen Arbeitsplatz bestimmt. ${ }^{60}$ Der zwischen negativ konnotiertem Studierzimmer des überholten Polyhistors und traditionellem Museum in der Tradition Petrarcas oszillierende Ort wird demnächst im Zuge der industriellen Revolution vielfältige neue Modelle wie Kontor, Büro, Labor entlassen, aber auch als heimeliges Arbeitszimmer erhalten bleiben. Auch Faust kennt diesen Typus, in dem ihm für einen Moment, in dem er den Prolog des Johannesevangeliums in ganz humanistischer Manier zu übersetzen versucht, die Ruhe des traditionellen Buchgelehrten vergönnt ist $(60,1194-1201)$ :

Ach wenn in unserer engen Zelle

Die Lampe freundlich wieder brennt,

Dann wird's in unserem Busen helle,

Im Herzen, das sich selber kennt.

Vernunft fängt wieder an zu sprechen,

Und Hoffnung wieder an zu blühn;

Man sehnt sich nach des lebens Bächen,

Ach! Nach des Lebens Quelle hin.

Die nächste Regieanweisung lautet entsprechend (61): «Er schlägt ein Volum auf und schickt sich an.»

\section{Literaturverzeichnis}

\section{Quellen}

Bullinger, H., Studiorum ratio - Studienanleitung, Bd. 1, hg. v. Peter Stotz, Zürich 1987.

Ficino, M., Three Books on Life: A Critical Edition and Translation with Introduction and Notes, hg. v. Carol V. Kaske, J. R. Clark, Binghampton / New York 1989.

Goethe, J. W., Faust. Texte, Bd. 1, hg. v. A. Schöne, Frankfurt a. M. 1977.

Ders., Werke, Hamburger Ausgabe, Bd. 9, hg. v. E. Trunz u. a., Hamburg 1955.

Machiavelli, N., Tutte le opere, hg. v. M. Martelli, Florenz 1971.

Petrarca, F., De vita solitaria. Buch I. Kritische Textausgabe und ideengeschichtlicher Kommentar, hg.v. K. A. Enenkel, Leiden / New York / Kopenhagen 1990.

Ders., De vita solitaria, hg. v. G. Martellotti, übers. v. A. Buffano, Mailand / Neapel 1955.

Ders., Epistulae metricae. Briefe in Versen, hg. und übers. v. E. Schönberg, O. Schönberg, Würzburg 2004.

Ders., Heilmittel gegen Glück und Unglück. De remediis utrisque fortunae. Lateinisch-deutsche Ausgabe in Auswahl, hg. v. E. Keßler, übers. und komm. v. R. Schottlaender, München 1988.

Ders., Prose, hg. v. G. Martellotti et al., Mailand / Neapel 1955.

${ }^{60}$ Eigler 2015, 13-15. 
Platter, F., Tagebuch (Lebensbeschreibung) 1536-1567, hg. v. V. Lötscher, Basel / Stuttgart 1976.

Rhenanus, B., Der Briefwechsel des Beatus Rhenanus, hg. v. A. Horawitz, K. Hartfelder, Leipzig 1886.

Schnur, H. C., Lateinische Gedichte Deutscher Humanisten, Stuttgart $1978^{2}$.

\section{Sekundärliteratur}

Anderson, J., Il risveglio dell'interesse per le Muse nella Ferrara del Quattrocento, in: A. Mottola Molfino, M. Natale (Hg.), Le Muse e il Principe. Arte di corte nel Rinascimento padano, Modena 1991, 165-86.

Aurnhammer, A., Petrarcas Katze. Geschichte des kätzischen Petrarkismus, Heidelberg 2005.

Bec, Chr., De Pétrarque á Machiavel; á propos d'un topos humaniste (le dialogue lecteur/livre), Rinascimento 16, 1976, 3-17.

Bertilli, S., Urbino: tra collezionismo e magia, in: A. Marchi (Hg.), Lo studiolo del Duca. Il ritorno degli uomini illustri alla corte di Urbino, Mailand 2015, 27-35.

Blanchard, W. S., Petrarch and the Genealogy of Asceticism, Journal of the History of Ideas 62, 3 2001, 401-423.

Brogsitter, K. O., Das hohe Geistergespräch. Studien zu humanistischen Vorstellungen einer zeitlosen Gesellschaft der großen Geister, Bonn 1958.

Celenza, Chr. S., Le studiolo à la Renaissance, in: Chr. Jacob (Hg.), Lieux de savoir. Espaces et communautés, Paris 2007, 371-391.

Eigler, U., Luxus, Lebensraum und Labyrinth. Bibliotheken in der griechischen und lateinischen Literatur, in: R. Kussl (Hg.), Antike Welt und Literatur. Einblicke, Analysen und Vermittlung im Unterricht, Speyer 2007, 9-26.

Ders., Libri peculiares? Der Codex als Ausdruck und Rahmen individueller Entfaltung, in: Th. Haye, J. Helmrath (Hg.), Codex im Diskurs, Wiesbaden 2014, 231-249.

Ders., Shre Ohren werden Augen machen`. Ruhe und Stille am Arbeitsplatz, in: Education permanente. Schweizerische Zeitschrift für Weiterbildung 4, 2015, 13-15.

Ders., De vita solitaria. Petrarca e la <reinvenzione〉 dello studioso, in: Humanistica 10, 2015, 85-92.

Ders., Natur auf Distanz: Die Landschaft im Arbeitszimmer, in: M. Kasper, M. Korenjak, R. Rollinger u. a. (Hg.), Entdeckungen der Landschaft: Raum und Kultur in Geschichte und Gegenwart, Wien 2017, 213-233.

Enenkel, K. A. E., Petrarca, Francesco, De vita solitaria, Buch I. Kritische Textausgabe und ideengeschichtlicher Kommentar, Leiden / New York / Kopenhagen 1990.

Fischer, K., Spinozas Leben, Werke und Lehre, Paderborn 2015 (Nachdruck des Erstdrucks von 1898).

Geiss, J., Zentren der Petrarca-Rezeption in Deutschland (um 1470-1525): Rezeptionsgeschichtliche Studien und Katalog der lateinischen Drucküberlieferung, Wiesbaden 2002.

Grünbein, D., Der cartesische Taucher. Drei Meditationen, Frankfurt a. M. 2008.

Hedeman, A. D., L’humanisme et les manuscrits enluminés: Jean Lebeégue et le manuscrit de Salluste de Genéve, Bibliothéque publique et universitarire, ms. 54., in: E. Taburet-Delahaye (Hg.), La création artistique en France autour de 1400. École du Louvre, Musée des Beaux-Arts de Dijon, Université de Bourgogne: Ecole du Louvre 7 et 8 juillet 2004: Musée des Beaux-Arts de Dijon, Université de Bourgogne 9 et 10 juillet 2000, Paris 2006, 449-464 [zitiert Hedemann 2006a]. 
DERs., Making the Past Present Visual Translation in Jean Lebégue’s 'Twin〉 Manuscripts of Sallust, in: G. Croenen, P. Ainsworth (Hg.), Patrons, Authors and Workshops. Books and Book Production in Paris around 1400, Louvain / Paris / Dudley 2006, 173-196 [zitiert Hedemann 2006b].

Hindermann, J., Orte der Inspiration in Plinius' Epistulae, Museum Helveticum 66, 2009, 223-231.

Karnein, A., Petrarca in Deutschland: Zur Rezeption seiner lateinischen Werke im 15. und 16. Jahrhundert, in: G. W. Weber (Hg.), Idee, Gestalt, Geschichte: Festschrift Klaus von See. Studien zur europäischen Kulturtradition, Odense 1988, 159-186.

LAzzi, G. Dalla cattedra allo studiolo: per l'iconografia del dotto nella miniatura del '400, Rara volumina, Bd. 8, 2001, 5-17.

Liebenwein, W., Studiolo. Die Entstehung eine Raumtyps und seine Entwicklung bis um 1600, Berlin 1977.

Minges, K., Das Sammlungswesen der frühen Neuzeit. Kriterien der Ordnung und Spezialisierung, Münster 1998.

Mitterauer, M., Warum Europa? Mittelalterliche Grundlagen eines Sonderwegs, München $2009^{5}$.

Pёснт, O., Zur Entstehung des «Hieronymus im Gehäus〉, Pantheon 20, 1963, 131-142.

Perrig, S., Am Schreibtisch großer Dichter und Denkerinnen. Eine Geschichte literarischer Arbeitsorte, Zürich 2011.

Porcher, J., Jean Lebégue, illustrateur de Salluste, Librarium 5, 1962, 159-170.

Rösler, W., Von der Klosterbibliothek zum Studiolo. Historische Anmerkungen zum «Lernort» Bibliothek, in: K. Westphal (Hg.), Orte des Lernens. Beiträge zu einer Pädagogik des Raumes unter Mitarbeit von Nicole Hoffmann, Weinheim 2007, 43-47.

SснӧN, E., Der Verlust der Sinnlichkeit oder die Verwandlungen des Lesers. Mentalitätswandel um 1800, Stuttgart 1987.

Schöne, A. Johann Wolfgang Goethe. Faust. Kommentare, Frankfurt am Main, 1999.

Stierle, K., Francesco Petrarca. Ein Intellektueller im Europa des 14. Jahrhunderts, München 2003. 